\title{
A SPINAL OSTEOBLASTOMA IN A CHILD, SITUATED IN THE VERTEBRAL BODY - CT AND MR IMAGING
}

\author{
Svjetlana MUJAGIĆ ${ }^{*}$, Maida KULJANIN², Asmir HRUSTIĆ 3
}

${ }^{1}$ Department of Radiology and Nuclear Medicine, ${ }^{2}$ Department of Pathology

Policlinic for laboratory diagnostic ${ }^{3}$ Department of Orthopedics of the University Clinical Center Tuzla Tuzla, Bosnia and Herzegovina

\footnotetext{
${ }^{*}$ Corresponding author: svjetlanamm@gmail.com Tel.: + 38735303585

Fax.: + 38735251456
}

Received: December 14, 2015 Accepted: January 22, 2016

Key words: Spinal osteoblastoma Vertebral body $\cdot \mathrm{CT} \cdot \mathrm{MR}$.

\section{Introduction}

Osteoblastomas are uncommon and benign osteoid-producing primary bone tumors, accounting for approximately $1 \%$ of all bone tumors, which frequently affect the long bones and the spine (1). Spinal osteoblastoma account for 30 to $40 \%$ of all osteoblastomas that occur in the second to third decades of life, and are slightly more frequent in males (2). Histologically, osteoblastomas show interconnecting trabecular bone and fibrovascular stroma larger than $2 \mathrm{~cm}$. Some authors believe that osteoblastomas and osteoid os-
Objective - To report a rare case of a lumbar spinal osteoblastoma involving only the vertebral body. Case report - A 10-year-old boy presented with low back and leg pain, difficulty walking, limping with his right leg, discreet scoliosis on the left side, and lumbar paraspinal muscle spasms. The Lasegue sign for both sides was positive at around 45 degrees. Babinski's sign and sensory changes were not noted. The boy was evaluated by X-ray, computed tomography (CT) and magnetic resonance imaging (MRI) of the lumbosacral spine. CT and MRI scans revealed an expansile lesion that involved the posterior part of the L4 vertebral body, sparing the posterior osseus elements of the L4 vertebra. The lesion extended into the spinal canal, with compression of the thecal sac and the left L5 nerve root. MR images showed osseus edema of the remainder of the L4 vertebral body, enhanced homogeneously. Peritumoral edema extended into the epidural soft-tissue. Total removal of the tumor was performed. The histopathological finding was an aggressive osteoblastoma. Conclusion - Spinal osteoblastomas are extremely rarely situated in the vertebral body alone. CT and MRI are very important methods for delineation of the location, osseous involvement, peritumoral edema and spread of the tumor in the epidural space, with the possible compression by the tumor of the neural elements of the vertebral canal. Total removal of the osteoblastoma is very important because some osteoblastomas may be aggressive, with a tendency to local recurrence and sometimes metastasis.

teomas belong to the same family of osteoblastic bone tumors, because they are clinically and histologically similar (3). In most of the literature they are described as distinct clinical entities. Spinal osteoblastomas usually arise within the posterior elements of the spine, while vertebral body involvement rarely occurs, especially alone (4). As osteoblastomas are locally aggressive tumors that can transform into osteosarcomas, they need to be well imaged by computed tomography (CT) and magnetic resonance imaging (MRI) for complete surgical removal. Also, 
MRI is able to determine intraosseous and soft tissue extension accurately, and distinguish the tumor from peritumoral edema in the bone and soft tissue (5).

We report a case of a lumbar spinal osteoblastoma in a child, situated only in the vertebral body, with rich CT and MRI findings.

\section{Case report}

A 10-year-old boy was admitted to our hospital with low back and leg pain over the previous month. The pain appeared a few days after falling from a chair, and was stronger after prolonged sitting or walking. The intensity of the pain increased over time. Walking was difficult. On examination, the boy limped discretely on his right leg. His right shoulder was slightly lowered, discreet scoliosis on the left and lumbar paraspinal muscle spasms were persistent. Lumbar spine movements were restricted. The Lasegue sign for both sides was positive, at around 45 degrees. Babinski's sign and sensory changes were not noted. Plain $x$-ray of the lumbosacral spine did not show any pathological findings. CT scans of the lumbosacral spine revealed an oval expansile lytic lesion that involved the posterior part of the L4 vertebral body. The adjacent bone of the vertebral body appeared to be sclerotic (Fig. 1). No visible fracture
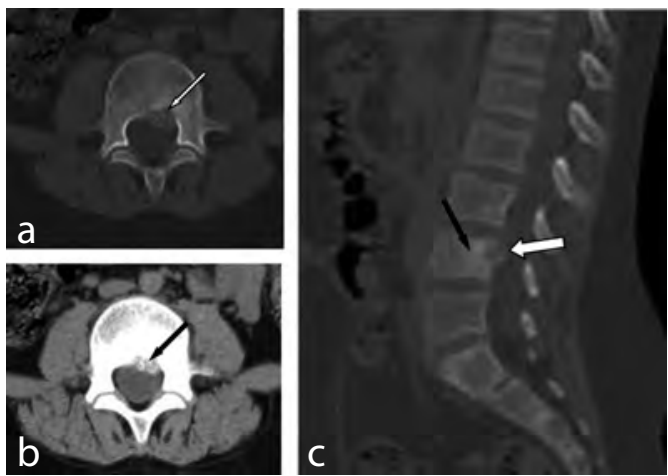

Fig. 1 The bone and soft window images from the axial CT scans (Fig. a, b) with sagittal reconstruction (Fig. c), showing the expansile lytic lesion that involves the posterior part of the L4 vertebral body (white arrows) within the surrounding sclerotic reactive bone (black arrow).
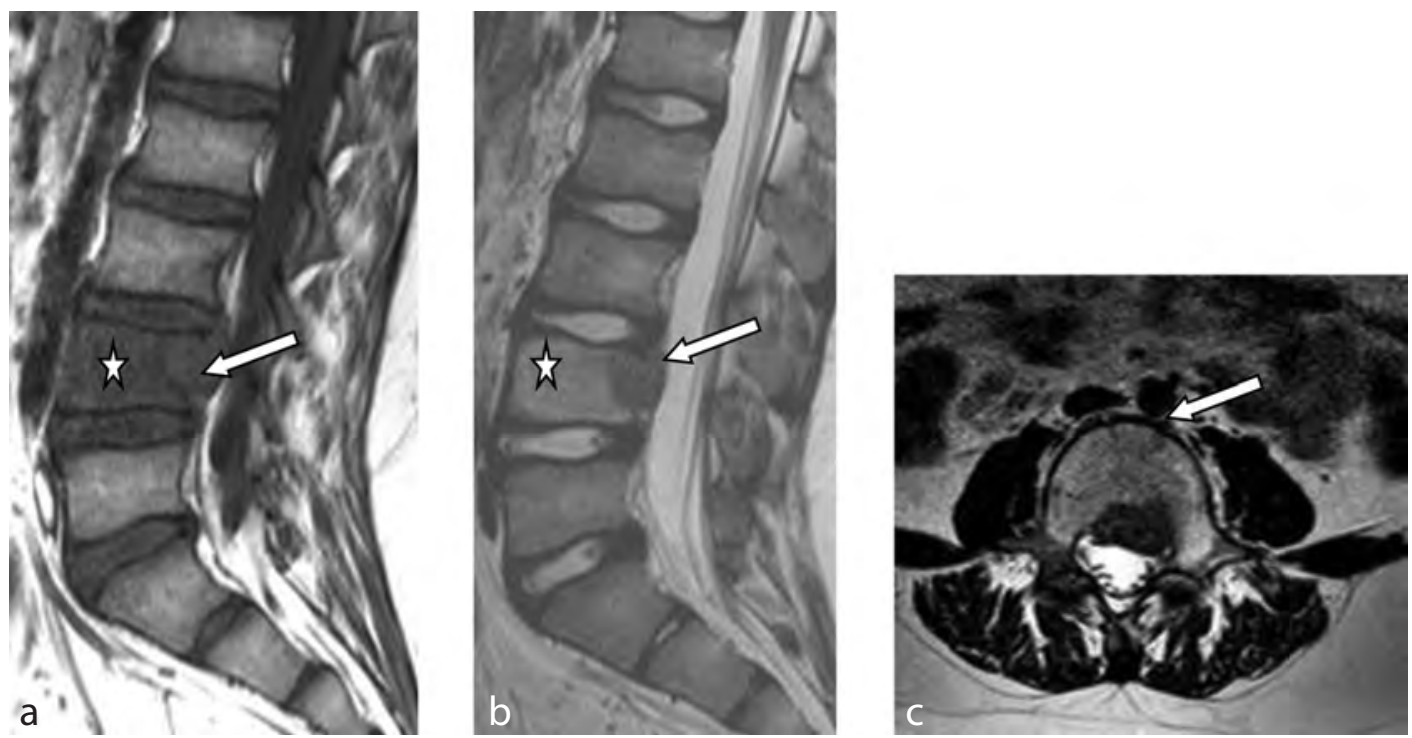

Fig. 2 The sagittal T1W/T2W (Fig. a, b) and axial T2W images (Fig. c) showing an expansile hypointense lesion in the posterior part of the L4 vertebral body extended into the spinal canal with compression of thecal sac and the left L5 nerve root (white arrows). Intense edema ("flare phenomenon") exists on the bone marrow of the rest of the L4 vertebral body (stars). The hypointense tumor is clearly distinguishable from the hyperintense edema on the T2W images (Fig. b, c). 

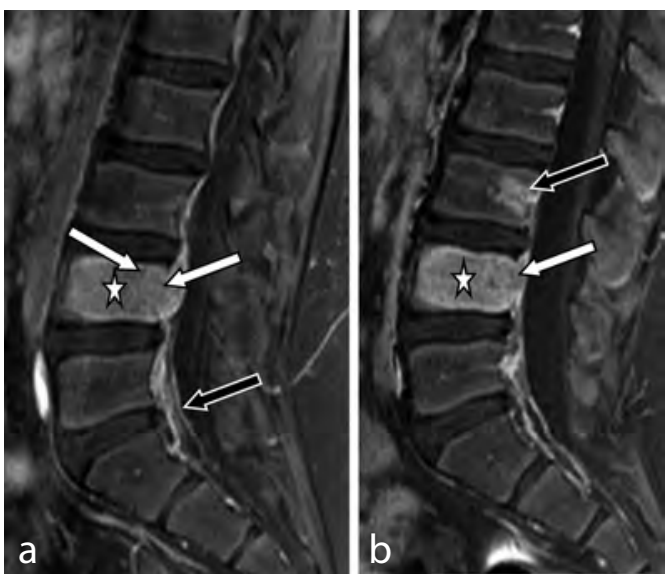

Fig. 3 The sagittal contrast-enhanced T1-weighted images showing the highly vascular tumor in the posterior part of the L4 vertebral body extended into the spinal canal with compression of thecal sac (white arrows). Intense enhancement of the edematous bone marrow of the rest of the vertebral body (stars) extending into the epidural soft-tissue and posterior part of the L3 vertebral body, round the basivertebral vein (black arrow). It is difficult to distinguish the tumor from the edema in the bone marrow.

lines were noted. MRI of the lumbar spine, on T1 and T2-weighted images, demonstrated a hypointense lesion, with a diameter of $15 \times 24 \times 17 \mathrm{~mm}$ (AP-LL-KK), and strong contrast enhancement, situated in the posterior part of the L4 vertebral body (Fig. 2, 3). The lesion extended into the spinal canal with compression of thecal sac and the left L5 nerve root (Fig. 2c, 3). The pedicle and lamina on both sides were spared (Fig. 1, 2c). Also, MR images showed osseous edema of the remainder of the L4 vertebral body, enhanced homogeneously, in the same way or even more than the tumor itself. Peritumoral edema extended into the epidural soft-tissue cranio-caudal and into the posterior part of the L3 vertebral body (Fig. 3).

Surgery was performed and the tumor was totally removed. The pathology report revealed the possibly aggressive osteoblast characteristics of the tumor (Fig. 4) and the diagnosis was aggressive osteoblastoma. Control MRI 6 months, one year and two years

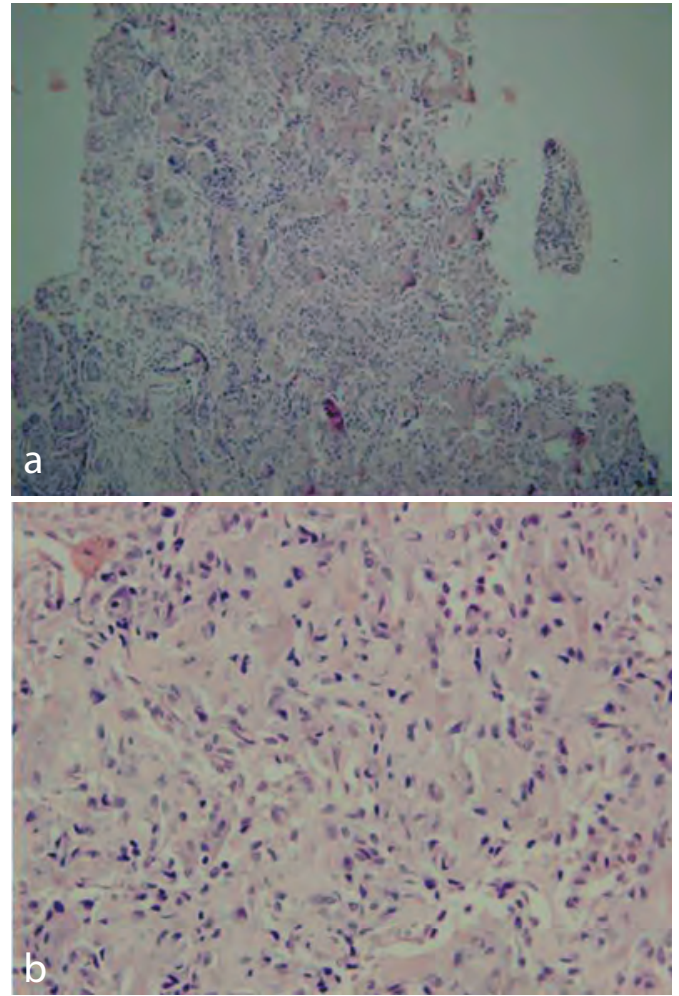

Fig. 4 Histopathology (hematoxylin - eosin staining $\times 10$ and $\times 40$ ). Parts of the tumor with loose to moderate cellular fibroblastic stroma, with a prominent vasculature and lacelike, focally irregular deposits of osteoid. Prominent rimming osteoblasts and multinucleated osteoclast-like giant cells are present (Fig. 4a). Most osteoblasts have characteristic epitheloid osteoblasts with abundant eosinophilic cytoplasm and an eccentric large, oval or round nucleus, with a prominent nucleolus (Fig. 4b). The mitotic rate is low, atypical mitosis is not seen and there are mild to moderate proliferation activities - with about 10\% Ki67 positive nuclei.

after surgery did not show any residual mass or recidive of the tumor. Annual MRI scans are recommended for the next few years to screen possible tumor recurrence.

\section{Discussion}

Osteoid osteomas and osteoblastomas are benign bone-forming tumors, which are histologically similar. According to the World Health Organization's definition, tumors $\leq 1$ 
$\mathrm{cm}$ in diameter are classified as osteoid osteoma, and those $\geq 2 \mathrm{~cm}$, as osteoblastoma. For tumors between $1 \mathrm{~cm}$ and $2 \mathrm{~cm}$, other criteria, such as the bone involved, the site, the presence of a nidus, and the presence of peripheral sclerosis, are used for diagnosis. The osteoid osteomas are mostly localized in the extremities, whereas the osteoblastoma cases involve the vertebral column and sacrum. The size of the nidus varies between 0.2 and $1.5 \mathrm{~cm}$ in osteoid osteoma cases, and the tumor size range is 1.3 to $10 \mathrm{~cm}$ in osteoblastomas (6). In osteoblastomas there is no nidus, characteristic of osteoid osteoma. Some osteoblastomas can be aggressive, with a tendency to local recurrence and sometimes metastasis (7). Osteoblastomas have the possibility of malignant transformation to osteosarcoma, but malignant transformation has only been reported in rare cases (8 to11). All reported cases of malignantly transformed osteoblastomas have shown conversion to osteosarcomas only in recurrent tumors. In the Mayo Clinic files, only one case of malignant transformation out of 108 cases of osteoblastoma has been recorded. The diagnosis of "malignancy" in an osteoblastoma is particularly difficult because of the aggressive behavior of some osteoblastomas (11). Osteoblastomas commonly affect the vertebral column. The cervical spine is the first and the lumbar spine is the second most commonly affected area. Most of these lesions arise within the posterior elements of the spine (55\%) with extension into the vertebral body (42\%) (4). Vertebral body involvement rarely occurs alone $(3 \%)$ but the vertebral body may be involved via tumor extension through the pedicle $(4,12,13,14)$. The vertebral body may be more commonly affected in the cervical spine (13). Frequently, osteoblastomas extend into the epidural space surrounding the nerve roots and the cord, leading to radiculopathy or cord compression (15).

On CT, osteoblastomas usually show a lesion larger than $1.5 \mathrm{~cm}$ in diameter, with a well-defined margin, central calcification of the matrix and cortical expansion, with demarcation by a thin bone shell (Fig. 1). On MRI, osteoblastomas are typically hypoto isointense on T1-weighted images, and isointense to hypointense on T2-weighted images, with areas of decreased intensity. The calcified portions of the tumor give rise to low T1 and T2 signals, and the uncalcified portion displays a moderately high T2 signal, with characteristic inhomogeneous contrast enhancement (Fig. 2, 3). The lesion may be associated with characteristic peritumoral edema in the bone and soft-tissues. This peritumoral edema, known as the "flare phenomenon", is a widespread inflammatory response surrounding an osteoblastoma, that leads to diffuse, reactive, inflammatory infiltration. The edema in the bone marrow enhances homogeneously, more than the tumor itself (4) (Fig. 3). In the present case, the lumbar osteoblastoma was situated only in the vertebral body, without involvement of the posterior elements of the spine (Fig. $1,2 c)$. Invasion into the epidural space, with compression on the left L5 root, was also present (Fig. 2c, 3). Classical CT and MRI findings were present with a strongly emphasized "flare phenomenon" (Fig. 1, 2, 3).

Clinically, osteoblastomas are differentiated from osteoid osteomas by dull, localized pain that does not respond to salicylates, associated neurologic symptoms and scoliosis, which is less frequent and generally on the opposite side to the tumor. Neurological symptoms in osteoblastomas are more frequent than in osteoid osteomas, due to epidural invasion by the tumor and compression on the nerve roots and spinal cord $(2,4,16$, 17). The treatment of choice for osteoblastoma is complete surgical resection. Radiotherapy and chemotherapy, either alone or together, may be useful in selected patients with recurrent, aggressive tumors, or in patients with a surgically unresectable disease (18). 


\section{Conclusion}

Spinal osteoblastomas are a rare tumor which may be aggressive, with a tendency to local recurrence and sometimes metastasis. The tumors usually arise within the posterior elements of the spine. Isolated vertebral body involvement, as in the present case, occurs extremely rarely. CT and MRI are very important methods for delineating the location and osseous involvement of the mass, peritumoral edema and the spread of the tumor to the epidural space, with possible compression on the neural elements of the vertebral canal. It is necessary to know all these facts for good preoperative planning for complete removal of the tumor. Since the recurrence rate of the tumor is relatively high, annual MRI scans are recommended for a few years after removal of the tumor.

Authors' contributions: Conception and design: SM; Acquisition, analysis and interpretation of data: SM; MK; AH; Drafting the manuscript:SM; Revising it critically for important intellectual content: SM.

Conflict of interest: The authors declare that they have no conflict of interest.

\section{References}

1. Cerase A, Priolo F. Skeletal benign bone-forming lesions. Eur J Radiol. 1998;27(1):S91-7.

2. Rodallec M, Feldy A, Larousserie F, Anract P, Campagna $R$, Babinet $A$, at al. Diagnostic imaging of solitary tumors of the spine: What to do and say?. Radiographics. 2008;28(4):1019-41.

3. De Souza Diaz L, Frost HM. Osteoid osteomaosteoblastoma. Cancer. 1974;33(4):1075-81.

4. Abdel Razek AA, Castillo M. Imaging appearance of primary bony tumors and pseudo-tumors of the spine. J Neuroradiol. 2010;37(1):37-50.

5. Ye J, Liu L, Wu J, Wang S. Osteoblastoma of the rib with CT and MR imaging: a case report and literature review. World Journal of Surgical Oncology. 2012;10:49.

6. Yalcinkaya U, Doganavsargil B, Sezak M, Kececi B, Argin M, Basdemir G, at al. Clinical and morphological characteristics of osteoid osteoma and osteo- blastoma: a retrospective single-center analysis of 204 patients. Ann Diagn Pathol. 2014;18(6):319-25.

7. Ozaki T, Liljenqvist U, Hillmann A, Halm H, Lindner N, Gosheger G, at al. Osteoid osteoma and osteoblastoma of the spine: experiences with $22 \mathrm{pa}-$ tients. Clin Orthop Relat Res. 2002;397:394-402.

8. Kunze E, Enderle A, Radig K, Schneider-Stock R. Aggressive osteoblastoma with focal malignant transformation and development of pulmonary metastases: a case report with a review of literature. Gen Diagn Pathol. 1996;141(5-6):377-92.

9. McLeod RA, Dahlin D, Beabout JW. The spectrum of osteoblastoma. AJR Am J Roentgenol. 1976;126(2):321-5.

10. Seki T, Fukuda H, Ishii Y, Hanaoka H, Yatabe S. Malignant transformation of benign osteoblastoma: a case report. J Bone Joint Surg Am. 1975;57(3):424-6.

11. Horvai A, Unni KK. Premalignant conditions of bone. Journal of Orthopaedic Science. 2006;11(4):412-23.

12. Samdani A, Torre-Healy A, Chou D, Cahill AM, Storm PB. Treatment of osteoblastoma at C7: a multidisciplinary approach. A case report and review of the literature. Eur Spine J. 2009;18(2):196-200.

13. Stavridis SI, Pingel A, Schnake KJ, Kandziora F. Diagnosis and treatment of a C2-osteoblastoma encompassing the vertebral artery. European Spine Journal. 2013;22(11):2504-12.

14. Lucas DR, Unni KK, McLeod RA, O’Connor MI, Sim FH. Osteoblastoma: clinicopathologic study of 306 cases. Hum Pathol. 1994;25(2):117-34.

15. Avadhanam PK, Vuyyur S, Panigrahi MK. A rare occurrence of osteoblastoma in a child. Journal of Pediatric Neurosciences. 2010;5(2):153-6.

16. Erlemann R. Imaging and differential diagnosis of primary bone tumors and tumor-like lesions of the spine. Eur J Radiol. 2006;58(1):48-67.

17. Murphey MD, Andrews CL, Flemming DJ, Temple HT, Smith WS, Smirniotopoulos JG. Primary tumors of the spine: radiologic pathologic correlation Radiographics. 1996;16(5):1131-58.

18. Berberoglu S, Oguz A, Aribal E, Ataoglu O. Osteoblastoma response to radiotherapy and chemotherapy. Med Pediatr Oncol. 1997;28(4):305-9. 